Original Paper

\title{
Long Noncoding RNA HOXA-AS2 Promotes Papillary Thyroid Cancer Progression by Regulating miR-520c-3p/S100A4 Pathway
}

\author{
Fada Xia Yong Chen Bo Jiang Xin Du Yao Peng Wenlong Wang \\ Wanze Huang Tiecheng Feng Xinying Li \\ Department of General surgery, Xiangya Hospital, Central South University, Changsha, China
}

\section{Key Words}

Hoxa-AS2 $\bullet$ LncRNA $・$ PTC $・ \operatorname{miR}-520 c-3 p \cdot S 100 A 4 \cdot C e R N A$

\begin{abstract}
Background/Aims: Thyroid cancer is one of the most prevalent endocrine tumors. The present study examined the effects of IncRNA HOXA cluster antisense RNA2 (HOXA-AS2) on the progression of papillary thyroid cancer (PTC), and explored the underlying molecular mechanisms. Methods: Quantitative real-time PCR was used to detect HOXA-AS2, miR-520c$3 p$ and S100 calcium-binding protein A4 (S100A4) expression. Furthermore, the effects of HOXA-AS2 silencing and overexpression on cell proliferation, migration, and invasion were assessed in PTC in vitro by CCK8 and transwell assay. Furthermore, bioinformatics online programs predicted and luciferase reporter assay were used to validate the association of HOXA-AS2 and miR-520c-3p in PTC. Results: We observed that HOXA-AS2 was up-regulated in PTC tissues. In vitro experiments revealed that HOXA-AS2 knockdown significantly inhibited cell growth in PTC in vitro and in vivo. Further functional assays indicated that HOXA-AS2 significantly promoted PTC cell migration and invasion by promoting EMT. Bioinformatics online programs predicted that HOXA-AS2 sponge miR-520c-3p at 3'-UTR with complementary binding sites, which was validated using luciferase reporter assay. HOXA-AS2 could negatively regulate the expression of miR-520c-3p in PTC cells. MiR-520c-3p was down-regulated in PTC tissues, and S100A4 was predicted as a downstream target of miR-520c-3p, which was confirmed by luciferase reporter assay. Conclusion: In summary, our results suggested that the HOXA-AS2/miR-520c-3p/S100A4 axis may play an important role in the regulation of PTC progression, which provides us with new insights into understanding the PTC.
\end{abstract}

(C) 2018 The Author(s)

Published by S. Karger AG, Basel

\begin{tabular}{ll}
\hline Xinying Li & Department of General surgery, Xiangya Hospital, Central South University \\
& No. 87 Xiangya Road, Changsha (China) \\
& E-Mail Jianguang1617@163.com
\end{tabular}




\section{Cellular Physiology Cell Physiol Biochem 2018;50:1659-1672 and Biochemistry \begin{tabular}{l|l} 
DOI: $10.1159 / 000494786$ & $\begin{array}{l}\text { (c) } 2018 \text { The Author(s). Published by S. Karger AG, Basel } \\
\text { www.karger.com/cpb }\end{array}$
\end{tabular} \\ Xia et al.: HOXA-AS2 and Papillary Thyroid Cancer}

\section{Introduction}

Thyroid cancers of follicular cell origin are the most common endocrine malignancies. The dominant histological type of thyroid carcinoma is papillary thyroid carcinoma (PTC) that accounts for $80-85 \%$ of all cases [1,2]. Although the overall 5-year survival rate of PTC exceeds the rate for most other cancers, the development of metastasis continues to be the most significant cause in thyroid cancer mortality [3]. The tumour invasion and metastases of PTC is a complex process involving the proliferation and detachment of tumour cells, breaking of the extracellular matrix, angiogenesis, hematogenous and lymphatic dissemination, invasion of the surrounding extracellular matrix, angiogenesis, vascular or lymphatic dissemination, and eventually, homing in distant tissues, and proliferation at novel sites that eventually results in metastatic tumors [4]. The molecular mechanism underlying PTC metastasis still remains elusive. Thus, it is important to identify genes involved in this process and develop new strategies to PTC treatment.

Long noncoding RNA (lncRNA) is a type of noncoding RNA greater than 200 nucleotides in length $[5,6]$. LncRNA could regulate the gene expression at the transcriptional or posttranscriptional level by acting as microRNA (miRNA) sponges [7]. Abnormal lncRNA expressions have been reported to be upregulated in various cancers, including PTC [8-10]. Recently, increasing evidence has shown that HOXA cluster antisense RNA 2 (HOXA-AS2), a lincRNA located between and antisense to the human HOXA3 and HOXA4 genes, promote several tumorigenic features including survival, proliferation and invasion [11-13]. However, the biological functions of IncRNA HOXA-AS2 in PTC and the related mechanisms are still unclear. In this study, we explored the role of HOXA-AS2 in the regulation of proliferation, migration and invasion of PTC.

Many studies have shown that lncRNAs can serve as a competing endogenous RNAs (ceRNAs) to play a post-transcriptional regulatory role in miRNA distribution on their targets [12]. miRNAs are a class of 21-25 nucleotide small non-coding RNA with no proteincoding potential. miRNAs have crucial roles in the regulation of gene expression through binding to the 3'-UTR of the target genes [14]. Recent studies have also demonstrated that the dysregulation of miRNAs is involved in a wide range of human cancers, including PTC. More and more studies had found that miR-520c-3p influences the biological progression of various cancer cells [15]. Recent study has demonstrated that miR-520c-3p negatively regulates EMT by targeting IL-8 to suppress the invasion and migration of breast cancer [16]. In addition, aberrant miR-520c-3p expression may lead to reduced IL-8 expression and promote the mesenchymal phenotype in breast cancer cells, thereby increasing invasive growth [17].

The present study aimed to explore the ceRNA mechanism of HOXA-AS2 though miR$520 c-3 p$ and revealed the functional relevance of miR-520c-3p and HOXA-AS2 in PTC. In this study, we found that upregulation of HOXA-AS2 in PTC tissues and cells were associated with increased cell viability and promotion of colony formation. Our analysis indicated that HOXA-AS2 functioned as a ceRNA to regulate the expression of S100 calcium-binding protein A4 (S100A4) by competing for miR-520c-3p binding and thereby promoting PTC growth.

\section{Materials and Methods}

Thyroid tissue samples

The paired samples used in this study $(n=128)$ consisted of tumor tissue and adjacent unaffected thyroid tissue from PTC patients collected at Department of General surgery, Xiangya Hospital, Central South University from January 2010 to June 2016 were collected. All cases were confirmed via pathological diagnosis. These patients did not receive the chemotherapy, radiotherapy or other treatments of thyroid cancer before operation. All the patients were pathologically confirmed and the tissues were collected immediately after they were obtained during the surgical operation, and then stored at $-80^{\circ} \mathrm{C}$ to prevent RNA loss. Written informed consent was obtained from all patients included in the study. 


\section{Cellular Physiology Cell Physiol Biochem 2018;50:1659-1672 \begin{tabular}{ll|l} 
and Biochemistry Published onlIne: 2 November 2018 & $\begin{array}{l}\text { (c) } 2018 \text { The Author(s). Published by S. Karger AG, Basel } \\
\text { www.karger.com/cpb }\end{array}$ \\
\hline
\end{tabular}}

Xia et al.: HOXA-AS2 and Papillary Thyroid Cancer

\section{Cell culture and reagents}

Four PTC cell lines (BHP5-16, TPC, K1 and BHP2-7) and a normal human thyroid epithelium cell line (Nthy-ori 3-1) were from the tumor cell bank of Chinese Academy of Sciences. Cells were cultured in RPMI 1640 (Invitrogen) supplemented with 10\% fetal bovine serum (Gibco) $100 \mathrm{U} / \mathrm{ml}$ penicillin, and $100 \mathrm{mg} /$ $\mathrm{ml}$ streptomycin (Invitrogen) in humidified air at $37^{\circ} \mathrm{C}$ with $5 \% \mathrm{CO} 2$. Hsa-miR-520c-3p mimics, hsa-miR520c-3p inhibitor, pcDNA3.1/HOXA-AS2 plasmid and negative control miRNA (miR-nc) were chemically synthesized by Shanghai Integrated Biotech Solutions Co., Ltd. (Shanghai, China).

\section{RNA extraction and $q R T-P C R$ assays}

Total RNA was extracted from tissues or cultured cells using TRIZOL reagent (Invitrogen). For qRTPCR, RNA was reverse transcribed to cDNA by using a Reverse Transcription Kit (Takara, Dalian, China). Real-time PCR analyses were performed with SYBR Premix Ex Taq (Takara, Dalian China). Results were normalized to the expression of GAPDH. The sequence of the primers were as following: HOXA-AS2 (Forward: 5'-CCCGTAGGAAGAACCGATGA-3', Reverse: 5'-TTTAGGCCTTCGCAGACAGC-3') and GAPDH (Forward: 5'-GGGAGCCAAAAGGGTCAT-3', Reverse: 5'-GAGTCCTTCCACGATACCAA-3'). The qRT-PCR assays were conducted on an ABI 7500 (Life Technologies, Carlsbad, CA, USA), and data collected with this instrument. Our qRT-PCR results were analyzed and expressed relative to threshold cycle (CT) values, and then converted to fold changes.

\section{Transfection}

Cells were seeded in 6-well dishes the night before to give $80-90 \%$ confluence for plasmid and $40-50 \%$ for miRNA at the day of transfection. The following day cells were transfected with miR-520c-3p mimics (50 $\mathrm{nM})$, miR-520c-3p inhibitor (100 nM), miR-nc (50 nM), inhibitor-nc (100 nM), pcDNA3.1/HOXA-AS2 (4.0 $\mu \mathrm{g})$ or pcDNA3.1 $(4.0 \mu \mathrm{g})$ respectively using Lipofectamine 2000 (invitrogen), according to the manufacturer's instructions. To perform effective lentivirus-mediated suppression of HOXA-AS2 in cells, the following HOXA-AS2 shRNA and scrambled control shRNA were inserted into the pLVX-tdTomato-Puro vector (Biowit, Shenzhen, China): HOXA-AS2 shRNA\#1 forward, 5'- GAGUUCAGCUCAAGUUGAACAUACA-3' and reverse, 5'UGUAUGUUCAACUUGAGCUGAACUC-3'; shRNA\#2 forward, 5'- AAACCUUGUAGAUAGCUUGAGCUGG-3' and reverse, 5'-CCAGCUCAAGCUAUCUACAAGGUUU-3'; shRNA\#3 forward, 5'-CAAGCUUGACAAGUUCAGCUCAA-3' and reverse, 5'- UUGAGCUGAACUCUUGUCAAGCUUG-3'; After transfection for 6 hours, the medium was replaced with normal culture medium. At $48 \mathrm{~h}$ post-transfection, cells were harvested for qRT-PCR analysis.

CCK-8 cell viability assay

After transfection, the cells mixed with $10 \mathrm{ml}$ of CCK-8 solutions per well and incubated for further $1 \mathrm{~h}$ at $37^{\circ} \mathrm{C}$. The amount of formazan dye generated by cellular dehydrogenase activity was measured for absorbance at $450 \mathrm{~nm}$ by a microplate reader (Molecular Devices, Sunnyvale, CA, USA). The optical density values of each well represented the survival/proliferation of cells.

\section{Matrigel invasion assay}

Cells were seeded ( $5 \times 104$ cells suspended in $0.5 \mathrm{ml}$ of no FBS media) in duplicate into 12 -well Corning BioCoat Matrigel Invasion Chambers with an $8 \mu \mathrm{m}$ pore size polyethylene terephthalate membrane. As a chemotaxis factor, FBS (10\%) was added to the lower compartment of the Matrigel Invasion Chambers. After 24 hours of incubation at $37^{\circ} \mathrm{C}$ in $5 \% \mathrm{CO} 2$ cells that had migrated through the pores were stained by JorVet DipQuick Stain assay and counted under a microscope (six slides per well; each experiment was repeated six times).

\section{Western blot analysis}

For protein extracts, cells were collected, washed with $1 \times$ PBS, resuspended with $2 \times$ SDS loading buffer (125 mM Tris pH 6.8, 4\% SDS, 20\% glycerol, $200 \mathrm{mM}$ dithiothreitol, $200 \mathrm{mM} \beta$-mercaptoethanol, $0.2 \% \mathrm{w} / \mathrm{v}$ bromophenol blue), and boiled for 10 minutes. Protein lysates were loaded onto $4-20 \%$ Criterion Tris-HCl precast gels (Bio-Rad), proteins were separated by electrophoresis (300V for 30 minutes), and subsequently transferred to $0.45 \mu \mathrm{m}$ polyvinylidene fluoride membranes. Membranes were incubated in $5 \%$ milk solution of Tris-buffered saline buffer containing 0.1\% Tween-20 (TBST) and incubated with primary antibodies diluted in $5 \%$ bovine serum albumin TBST solution containing $0.1 \%$ sodium azide overnight at 


\section{Cellular Physiology Cell Physiol Biochem 2018;50:1659-1672 \begin{tabular}{ll|l} 
and Biochemistry & $\begin{array}{l}\text { DOl: 10.1159/000494786 } \\
\text { Publisned online: } 2 \text { N November } 2018\end{array}$ & $\begin{array}{l}\text { O 2018 The Author(s). Published by S. Karger AG, Basel } \\
\text { www.karger.com/cpb }\end{array}$ \\
\cline { 1 - 3 }
\end{tabular}}

Xia et al.: HOXA-AS2 and Papillary Thyroid Cancer

$4^{\circ} \mathrm{C}$. Membranes were washed in TBST and probed with secondary antibodies. Following the final wash in TBST, membranes were incubated in ECL Western blotting detection reagents (GE Healthcare) and exposed to film (Denville). Antibodies used were anti-S100A4 (1:1000; ab124805; Abcam Inc., Cambridge, MA, USA), anti-E-cadherin (1:1000; ab40772; Abcam Inc., Cambridge, MA, USA), anti-N-cadherin (1:1000; ab76057; Abcam Inc., Cambridge, MA, USA), anti-vimentin (1:1000; ab8978; Abcam Inc., Cambridge, MA, USA) and anti-GAPDH (1:1000; \#517; Cell Signaling Technology, Beverly, MA, USA) primary and secondary antibodies and detected with Image Acquisition using Image Quant ${ }^{\mathrm{TM}}$ LAS 4000 (GE Healthcare Life Sciences, Michigan, USA).

\section{Tumor formation assay}

Female BALB/c nude mice were purchased at 5-6 weeks of age from Beijing Vital River Laboratory Animal Technology Co., Ltd. For xenograft models, $5 \times 105$ cells transfected with HOXA-AS2 shRNA as well as negative control (GenePharma) were subcutaneously injected in the bottom right back of BALB/c nude mice respectively. Tumor growth was examined every 3 days when the implantations were starting to grow bigger. After 4 weeks the mice were sacrificed, necropsies were performed and the tumor tissues were harvested. Tumor volumes were calculated as described previously.

\section{RNA immunoprecipitation (RIP) assay}

The RIP assay was performed to explore the interaction between HOXA-AS2 and miR-520c-3p by using EZ-Magna RIP RNA-binding protein immunoprecipitation kit (Millipore). Cells were lysed, and the cell lysis was then incubated with anti-human Ago2 antibodies (Millipore) coated on magnetic beads in RIP buffer. Input and normal IgG were used as controls. The precipitated RNAs were isolated and reverse transcribed in cDNA to analyze HOXA-AS2 and miR-520c-3p level using qRT-PCR.

\section{Luciferase reporter assays}

StarBase v2.0 (http://starbase.sysu.edu.cn/) was used to predict the binding sites between LncRNAHOXA-AS2 and miR-520c-3p. The luciferase reporter assays were carried out with the help of the Dualluciferase Reporter Assay System (Promega, Madison, WI, USA). The wide-type HOXA-AS2 or mutant HOXA-AS2 that had the predicted miR-520c-3p binding site was established and integrated into a pmir-GLO Dual-luciferase vector to form the pmirGLO-HOXA-AS2-wild type (HOXA-AS2-wt) or pmirGLO- HOXA-AS2mutant (HOXA-AS2-mut) reporter vector. Cotransfection of HOXA-AS2-wt or HOXA-AS2-mut was carried out with miR-520c-3p mimics or negative control into cells with the use of Lipofectamine 2000. Subsequent to transfection for a period of 48 hours, the luciferase activities were measured in accordance with the guidelines of the manufacturer. In the same manner, pmirGLO-S100A4-wild type (S100A4-wt) or pmirGLOS100A4-mutant (S100A4-mut) were constructed, together with cotransfecting with miR-520c-3p mimics or negative control into HEK293T cells. 48 hours following the transfection, the relative luciferase activities were detected.

\section{Immunohistochemistry}

For each patient sample, three paraffin sections of $5 \mu \mathrm{m}$ were prepared, one for hematoxylin and eosin (HE) staining and the other two for immunohistochemical staining. PBS instead of primary antibodies was used for negative control, and the breast cancer tissue was used for positive control. Sections were dewaxed using xylene, followed by hydration with ethanol solutions and addition of EDTA for antigen retrieval. Later, sections were blocked with normal goat serum for $30 \mathrm{~min}$ to eliminate non-specific binding. Sections were incubated with primary antibody against S100A4 (Abcam, Cambridge, UK). Sections were then incubated with biotin-labeled secondary antibodies for $30 \mathrm{~min}$ at room temperature, followed by staining with diaminobenzidine (DAB). Finally, the sections were counterstained with hematoxylin. The result of staining was determined by two doctors who did not know the clinical condition of patients. The proportions of positive cells of $0,1-5,6-25,26-75$, and 76-100 \% were assigned with scores of $0,1,2,3$, and 4 , respectively. Scores of $0-2$ were considered as negative expression, and scores of 3-4 were considered as positive expression. 


\section{Cellular Physiology Cell Physiol Biochem 2018;50:1659-1672 and Biochemistry \begin{tabular}{l|l} 
DOI: $10.1159 / 000494786$ & $\begin{array}{l}\text { @ } 2018 \text { The Author(s). Published by S. Karger AG, Basel } \\
\text { www.karger.com/cpb }\end{array}$
\end{tabular} \\ Xia et al.: HOXA-AS2 and Papillary Thyroid Cancer}

Statistical analysis

The presentation of the data has been made as the mean \pm SD from at least three sovereign experiments. Statistical analyses were carried out with the use of SPSS 18.0 software (SPSS, Chicago, IL, USA), together with generating the graphs using GraphPad Prism 6.0 (GraphPad Prism, San Diego, CA, USA). The relationship between HOXA-AS2 expression and clinical characteristics of patients was evaluated with the application of the chi-squared test. Analyses were carried out for the dissimilarities between groups through the application of Student's t test, one-way ANOVA analysis and Pearson's correlation analysis. P < 0.05 was considered statistically significant.

\section{Results}

LncRNA-HOXA-AS2 was up-regulated in human PTC tissues and cell lines

To determine the function of HOXA-AS2 during tumorigenesis of PTC, we first detected the expression of HOXA-AS2 in tumor tissues and adjacent normal tissues from 128 patients suffering from PTCs. Fig. 1A, qRT-PCR analysis displayed significantly higher levels of HOXAAS2 in PTC tissues compared to normal tissues. We found that high expression of HOXAAS2 was corrected with distant metastasis $\left({ }^{*} P<0.05\right.$, Fig. 1B). To investigate the roles of HOXA-AS2in PTC, we examined the correlation between clinical characteristics and HOXAAS2 expression in 128 PTC patients. We used the mean expression level of HOXA-AS2 in cancerous tissues to dichotomise the PTC cases. Univariate analysis showed that high HOXAAS2 expression group displayed a higher incidence of increased lymph node metastasis $(\mathrm{P}=0.001)$, multifocality $(\mathrm{P}=0.005)$ and advanced $\mathrm{TNM}$ stage $(\mathrm{P}=0.020)$. However, no significant differences were observed with regard to age, gender, or T stage (Table 1).

Furthermore, to confirm the upregulation of HOXA-AS2 in PTC cell lines, we performed qRT-PCR analysis to detect the HOXA-AS2 expression in 4 PTC cell lines (BHP5-16, TPC, K1, BHP2-7) and a normal human thyroid cell line Nthy-ori 3-1. As illustrated in Fig. 1C, HOXAAS2 mRNA expression level was significantly higher in four PTC cell lines as compared with Nthy-ori 3-1 $(P<0.01$, respectively). BHP5-16 and K1 cells showed the higher expression of HOXA-AS2, in relation to TPC and BHP2-7 cells. To analyze the role of HOXA-AS2 in PTC, BHP516 and K1 cells were stably transfected with HOXA-AS2 shRNAs (respectively shHOXA-AS2 \#1, shHOXA-AS2 \#2, or shHOXA-AS2 \#3) or empty vectors (sh-NC). To enhance HOXA-AS2 expression in PTC cells, a pcDNA3.1/HOXA-AS2 vector was constructed and transfected into TPC cells respectively. The cells transfected with pcDNA3.1 plasmid were acted as a control.

We detected HOXA-AS2 expression at $48 \mathrm{~h}$ post-transfection by qRT-PCR analysis to analyze efficiency and revealed that HOXA-AS2 expression was significantly decreased in BHP5-16 and K1 cells, and HOXA-AS2 expression was significantly increased in TPC cells, as compared with control group respectively (Fig. 1D-E). Moreover, shHOXA-AS2 \#3 had higher efficiency of interference than shHOXA-AS2 \#1 and \#2 group (Fig. 1D), so we chose shHOXAAS2 \#3 subsequently for the following experiments.

\section{HOXA-AS2 regulates PTC progression in vitro and in vivo}

To evaluate the biological effects of HOXA-AS2 in PTC cells, we compared the proliferation, migration and invasion ability of BHP5-16-shHOXA-AS2 \#3 and K1-shHOXA-AS2 \#3 cells with control cells. CCK-8 assays revealed that silencing of HOXA-AS2 expression significantly suppressed cell proliferation of BHP5-16 and K1 cells compared with the respective controls $(P<0.01$, Fig. $1 \mathrm{~F}$ and Fig. 2A). Whereas, cell proliferation was significantly increased in TPC cells infected with pcDNA3.1/HOXA-AS2 vector compared with negative controls $(P<0.01$, Fig. 2B).

The above in vitro data showed that HOXA-AS2 functioned as a tumor oncogene in PTC cell lines. To further determine the effects of HOXA-AS2 on tumorigenesis in vivo, BHP516 cell lines transfected with either empty vector or shHOXA-AS2 \#3 were subcutaneously implanted into nude mice. As expected, deregulation of HOXA-AS2 substantially attenuated the growth of tumors in nude mice as early as 4 weeks $(P<0.01$, Fig. 2C-D). Subsequently, 


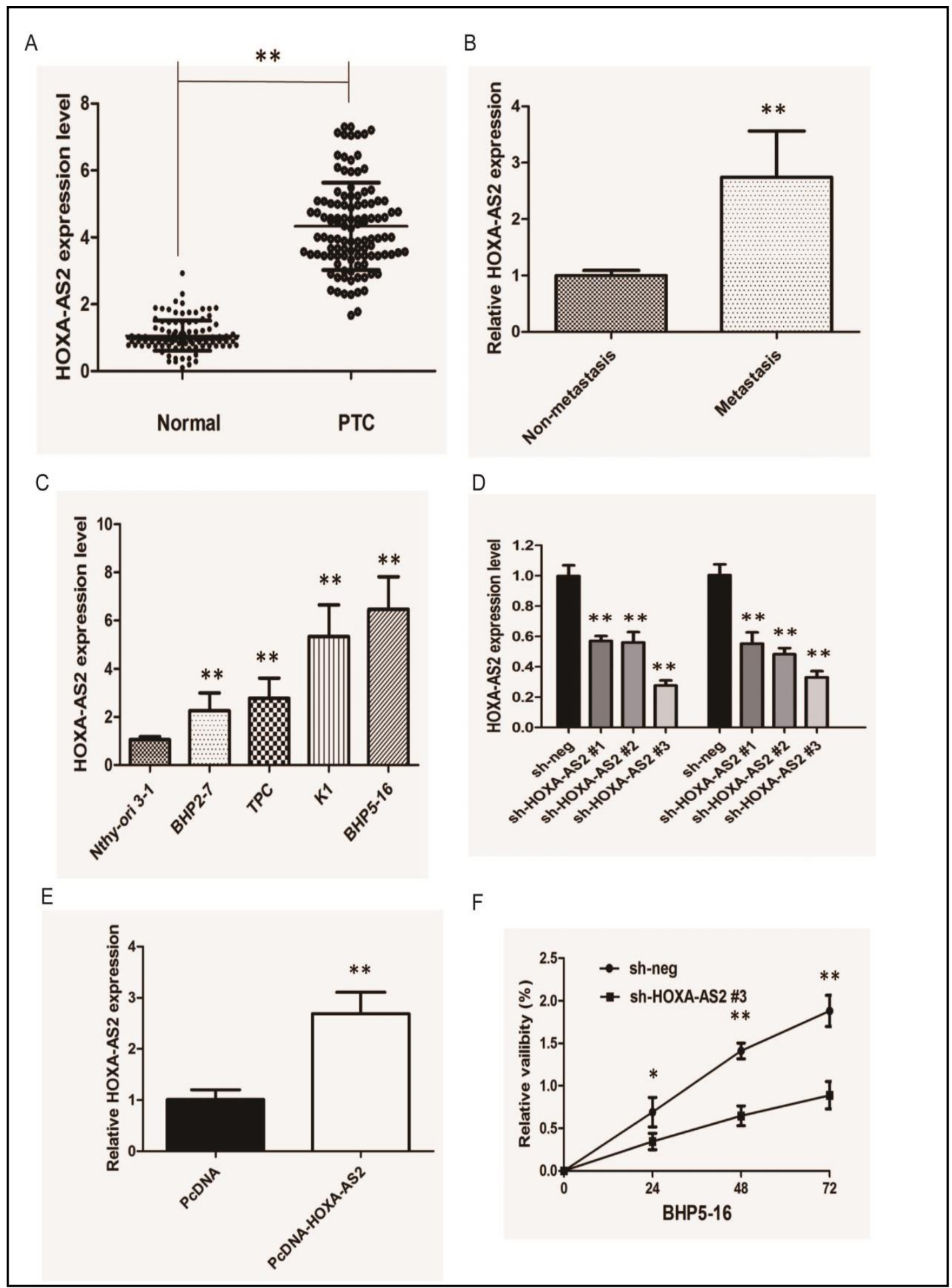

Fig. 1. A: qRT-PCR showing expression level of HOXA-AS2 in PTC tissues and adjacent noncancerous tissues; B: qRT-PCR showing expression level of HOXA-AS2 in metastasis PTC tissues; C: qRT-PCR showing expression level of HOXA-AS2 in PTC cell lines; D: HOXA-AS2 shRNAs was used to enhance efficiency of HOXA-AS2 knockdown in BHP5-16 and K1 cells; E: ectopic overexpression of HOXA-AS2 by transfecting TPC cell lines with pcDNA-HOXA-AS2 expression vector; F: CCK8 assay showing knockdown of HOXA-AS2 inhibited cell proliferation of BHP5-16 cells; All tests were at least performed three times. Data were expressed as mean $\pm \mathrm{SD} .{ }^{*} \mathrm{P}<0.01$. 
the weights of the tumors derived from BHP5-16-shHOXA-AS2 \#3 cells were clearly lower than those from NC cells after the period of 5 weeks post-implantation $(P<0.01$, Fig. 2E). The average expression level of HOXA-AS2 in sh-HOXA-AS2\#3 group was lower than that in empty group $(P<0.01$, Fig. $2 \mathrm{~F})$.

HOXA-AS2 promotes PTC cell migration and invasion via regulating EMT

To further confirm the effect of HOXA-AS2 on PTC cell migration and invasion, we investigated cell migration and invasion by transwell assay. The results showed that the migratory and invasive capacities of BHP5-16 and K1 cells dramatically decreased after deregulation of HOXAAS2 $(P<0.01$, Fig. 3A-B $)$. Furthermore, the transwell
Table 1. Clinical characteristics of 128 PTC patients according to HOXA-AS2 expression levels

\begin{tabular}{|c|c|c|c|c|}
\hline \multirow{3}{*}{ Characteristics } & \multirow{3}{*}{$\begin{array}{l}\text { Low } \\
(n=62)\end{array}$} & HOXA-AS2 & \multicolumn{2}{|l|}{ expression } \\
\hline & & & High & $P$ \\
\hline & & & $(n=66)$ & \\
\hline \multicolumn{5}{|l|}{ Age, $y$} \\
\hline$\geq 45$ & \multirow{2}{*}{30} & & \multirow{2}{*}{34} & \multirow{2}{*}{0.842} \\
\hline Gender & & & & \\
\hline Female & 44 & & 52 & 0.382 \\
\hline \multicolumn{5}{|l|}{ T-stage } \\
\hline T2-4 & 12 & & 20 & 0.325 \\
\hline \multicolumn{5}{|c|}{ Lymph node metastasis } \\
\hline Yes & 2 & & 35 & 0.001 \\
\hline \multicolumn{5}{|c|}{ TNM stage group } \\
\hline $\mathrm{II-IV}$ & 6 & & 20 & 0.020 \\
\hline \multicolumn{5}{|l|}{ Multifocality } \\
\hline Yes & 6 & & 30 & 0.005 \\
\hline
\end{tabular}

Fig. 2. A: CCK8 assay showing knockdown of HOXA-AS2 inhibited cell proliferation of $\mathrm{K} 1$ cells; B: CCK8 assay showing overexpression of HOXA-AS2 promoted cell proliferation of TPC cells; C-D: HOXA-AS2 knockdown inhibits PTC tumour growth in vivo. The tumour volume curve of nude mice was analysed; E:The tumour weights of nude mice were measured; F: The expression level of HOXA-AS2 in tumours of nude mice was detected by qRT-PCR. ${ }^{*} \mathrm{P}<0.05,{ }^{* *} \mathrm{P}<0.01$.
A

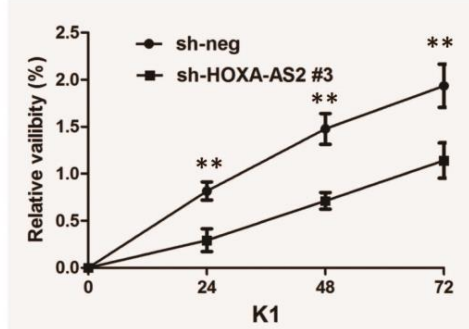

C
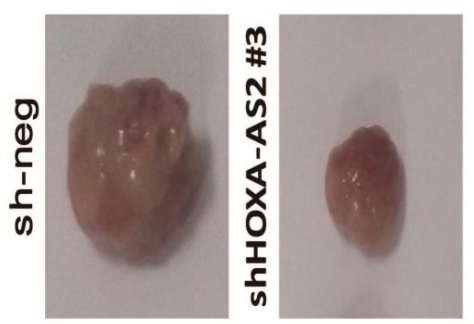

E

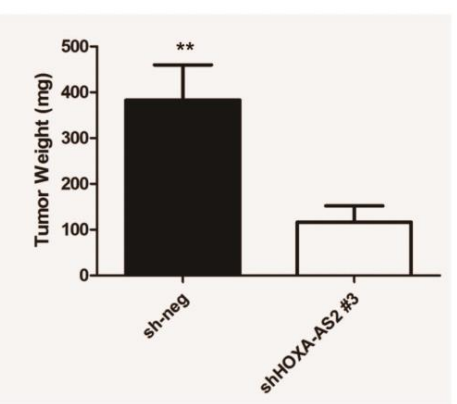

B

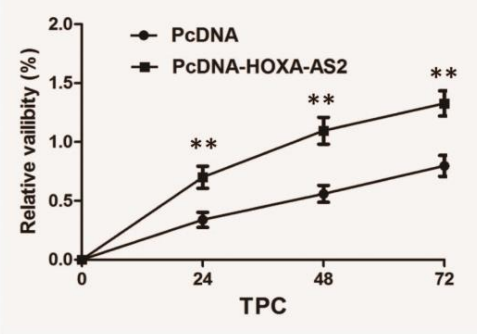

D
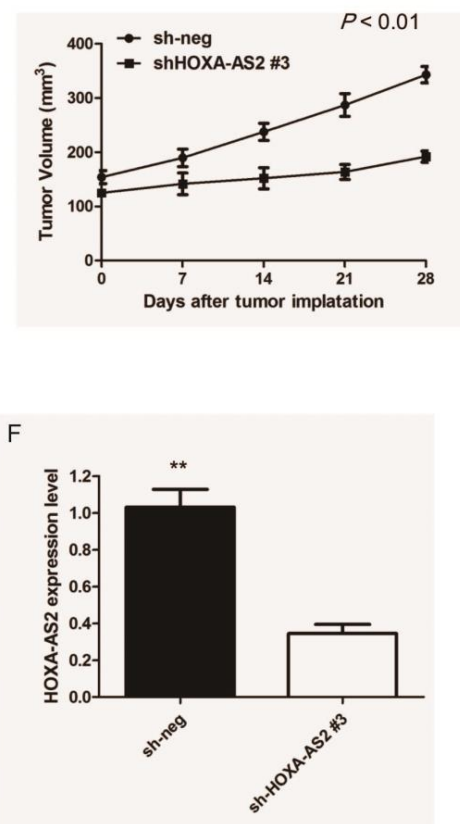


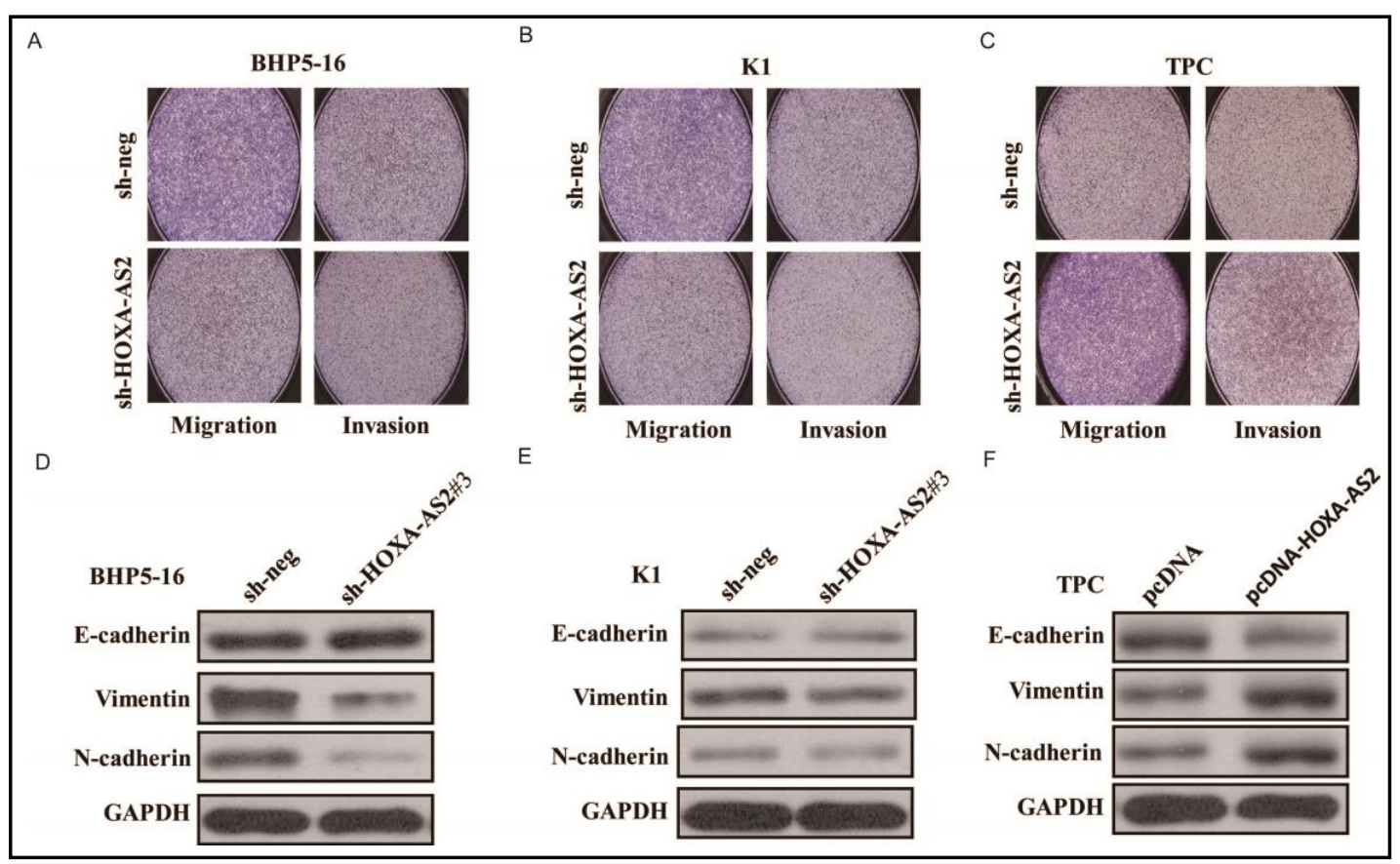

Fig. 3. A: Inhibition of migration and invasion of BHP5-16 cells transfected with shHOXA-AS2 \#3; B: Inhibition of migration and invasion of $\mathrm{K} 1$ cells transfected with shHOXA-AS2 \#3; C: Overexpression of HOXA-AS2 treatment significantly promoted the migration capacity of TPC cells; D: Knockdown of HOXAAS2 reverses EMT in BHP5-16 cells; E: Knockdown of HOXA-AS2 reverses EMT in K1 cells; F: Overexpression of HOXA-AS2 promotes EMT in TPC cells.

assay showed that overexpression of HOXA-AS2 treatment significantly promoted the migration and invasive capacity of TPC cells compared to NC $(P<0.01$, Fig. $3 C)$, suggesting that HOXA-AS2 could promote PTC cell migration and invasion.

We next examined whether silencing HOXA-AS2 expression inhibited mesenchymal features. As expected, the mesenchymal marker Vimentin and $\mathrm{N}$-cadherin were reduced in BHP5-16 and K1 cells after transfection of shHOXA-AS2 \#3. The expression of E-cadherin increased in both cells (Fig. 3 C-D). Overexpression of HOXA-AS2 treatment significantly decreased the expression of E-cadherin, and increased Vimentin and N-cadherin expression in TPC cells (Fig. 3 E). In conclusion, our results suggest that inhibition of HOXA-AS2 decreased the ability of migration and invasion of PTC cells, and that this effect likely relies on reversing the EMT process.

The interaction between HOXA-AS2 and miR-520c-3p in PTC cells

To investigate the effect of HOXA-AS2 on the expression of miRNAs, BHP5-16 cells were transfected with either empty vector or shHOXA-AS2 \#3 for 48 hrs. Hierarchical clustering of differentially expressed miRNAs is shown in Fig. 4A. Then, we applied the online software starBase v2.0. to predict the miRNAs that interacted with HOXA-AS2. The bioinformatics analysis revealed potential combination of HOXA-AS2 and miR-520c-3p, the putative binding sites as shown in Fig. 4B.

To verify whether HOXA-AS2 could directly interact with miR-520c-3p, we constructed luciferase vectors containing wild type or mutant binding sites of HOXA-AS2 in miR-520c-3p and performed luciferase reporter assay. As shown in Fig. 4C, miR-520c-3p overexpression significantly reduced the luciferase activity of pmirGLO-HOXA-AS2 reporter but had no obvious effect on their mutant reporters (pmirGLO-HOXA-AS2-mut). In a further RIP experiment, HOXA-AS2 and miR-520c-3p simultaneously existed in the production precipitated by anti-AGO2 (Fig. 4D), suggesting that miR-520c-3p is HOXA-AS2-targeting miRNA. 


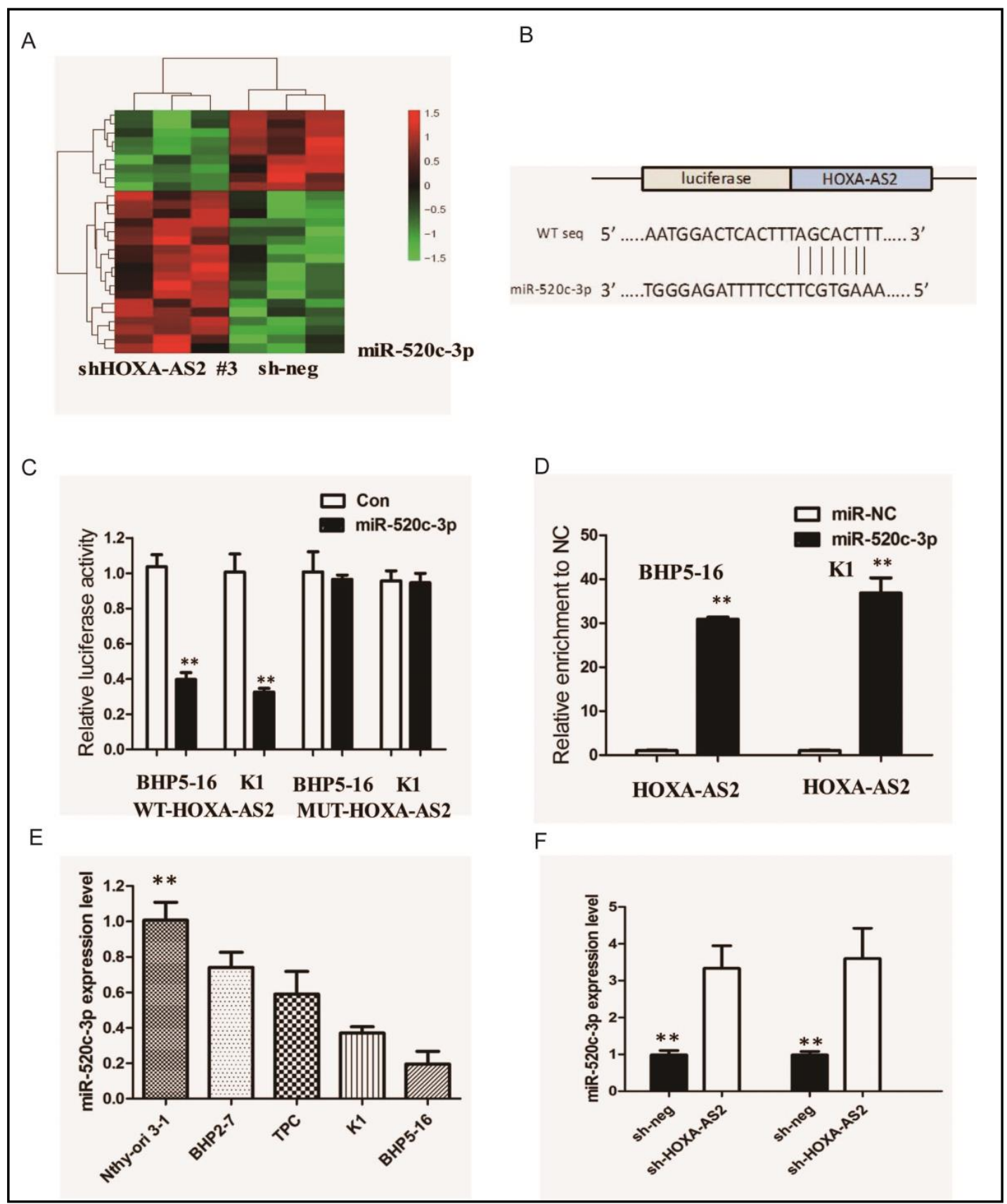

Fig. 4. A: BHP5-16 cells were transfected with shHOXA-AS2 \#3 for $48 \mathrm{hrs}$. Hierarchical clustering revealed systematic variations in the expression of miRNAs. Numerous differentially expressed miRNAs between control and shHOXA-AS2 \#3 transfected BHP5-16 cells are shown on a scale from green (low) to red (high); B: StarBase v2.0 results showing the sequence of HOXA-AS2 with highly conserved putative miR-520c-3p binding sites; C: The wild-type or mutant miR-520c-3p-binding sites in HOXA-AS2 were inserted into pMIRreport luciferase vector. Luciferase activity was detected in PTC cells co-transfected with miR-520c-3p or negative control (miR-control) and reporter plasmids containing WT-HOXA-AS2 (wild type) or MUT-HOXAAS2 (mutant type). The normalized luciferase activity in the miR-control group was used as the relative luciferase activity; D: HOXA-AS2 and miR-520c-3p simultaneously existed in the production precipitated by anti-AG02; E: Expression levels of miR-520c-3p in different PTC cell lines were determined by qRT-PCR; F: Silencing of HOXA-AS2 increased the expression level of miR-520c-3p in BHP5-16 and K1 cells; All tests were at least performed three times. Data were expressed as mean $\pm \mathrm{SD}$. ${ }^{*} \mathrm{P}<0.05,{ }^{* *} \mathrm{P}<0.01$. 


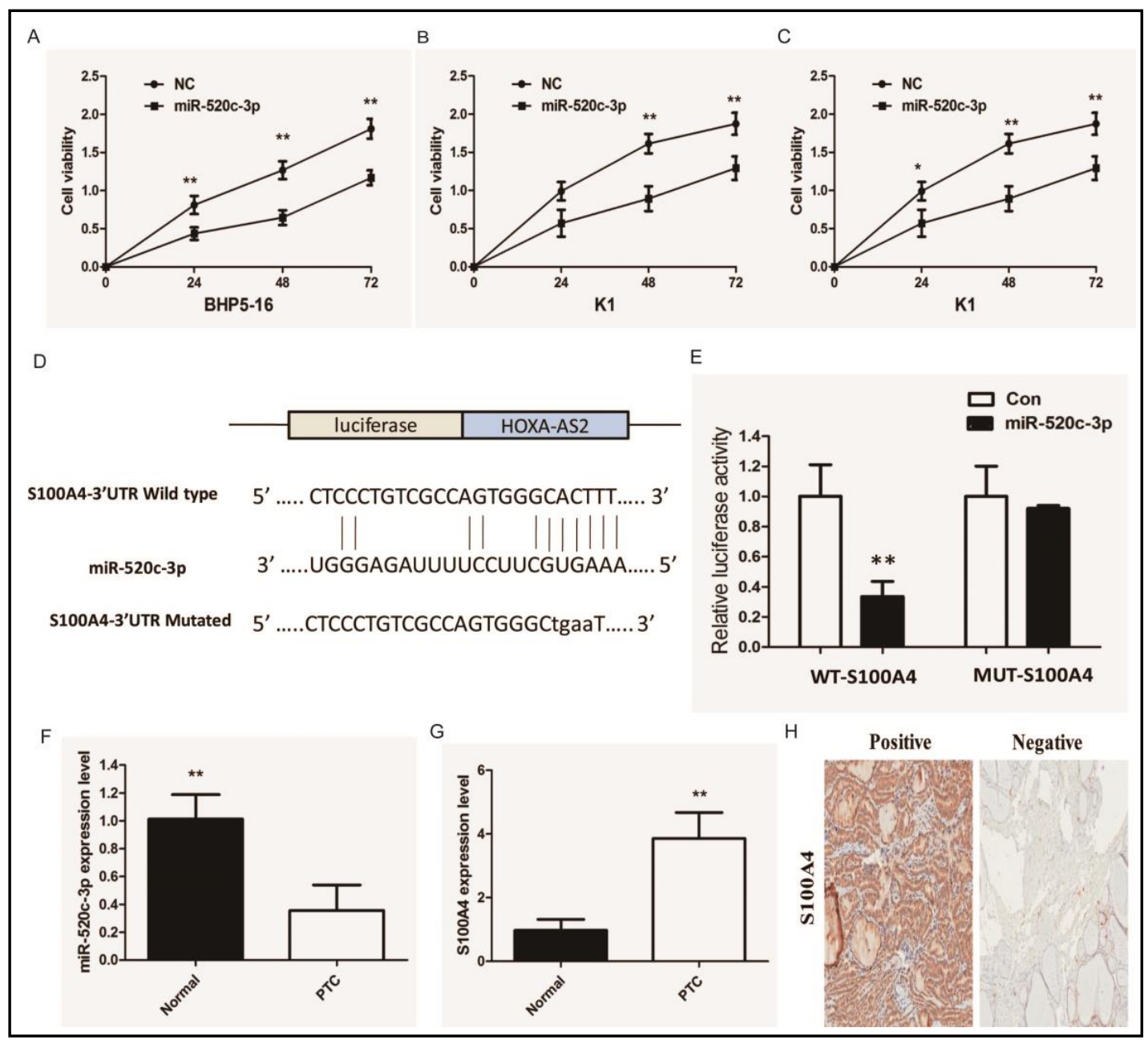

Fig. 5. A: CCK8 assay showing miR-520c-3p mimic inhibited cell proliferation of BHP5-16 cells; B: CCK8 assay showing miR-520c-3p mimic inhibited cell proliferation of K1 cells; C: CCK8 assay showing miR-520c$3 p$ inhibitor promoted cell proliferation of TPC cells; D: Bioinformatics analysis revealed the predicted binding sites between S100A4 and miR-520c-3p; E: Luciferase reporter assay demonstrated miR-520c3p mimics significantly decreased the luciferase activity of S100A4-wt in HEK293T cells; F: miR-520c-3p expression was significantly downregulated in PTC tissues compared with adjacent normal tissues by qRTPCR analysis; G: S100A4 expression was significantly upregulated in PTC tissues compared with adjacent normal tissues by qRT-PCR analysis; H: IHC analysis were performed to examine the expression levels of $\mathrm{S} 100 \mathrm{~A} 4$ in PTC tissues and adjacent normal tissues; ${ }^{*} \mathrm{P}<0.01$.

Next, we measured the levels of miR-520c-3p expression in various PTC cell lines. As shown in Fig. 4E, the expression of miR-520c-3p was obviously decreased in BHP5-16 and K1 cells, indicating the opposite result to HOXA-AS2 expression. To explore the regulatory relationship between HOXA-AS2 and miR-520c-3p, BHP5-16 and K1 cells were transfected with either empty vector or shHOXA-AS2 \#3, and qRT-PCR was used to detect the expression level of miR-520c-3p. The results showed that silencing of HOXA-AS2 increased the expression level of miR-520c-3p in BHP5-16 and K1 cells $(P<0.01$, Fig. 4F).

miR-520c-3p inhibits PTC progression by targeting S100A4

To investigate the role of miR-520c-3p on PTC carcinogenesis, miR-520c-3p mimics or inhibitor was transfected into PTC cell lines and the proliferation curves were performed using CCK8 assays. Our results showed that miR-520c-3p overexpression markedly inhibits the cell growth in both BHP5-16 and K1 cells when compared with cells transfected with 
miR-nc, whereas the TPC cells transfected with miR-520c-3p inhibitor grew at a dramatically higher rate as compared with controls (Fig. 5A-C). Collectively, these data indicate that miR520c-3p can inhibit PTC cells proliferation, which inversely correlates with the effects of HOXA-AS2 in PTC cells.

To identify potential target genes of miR-520c-3p, we searched for candidate genes using TargetScan6.2 and miRBase miRNA databases. Bioinformatics analysis showed that miR-520c-3p directly targets S100A4 (Fig. 5D). To confirm the predictions, a wild type or mutant S100A4 3' UTR luciferase reporter vector was conducted. S100A4-wt or S100A4-mut was co-transfected with miR-520c-3p mimics or negative control into HEK293T cells. The relative luciferase activity was remarkably reduced in cells co-transfected with the S100A4wt luciferase reporter and miR-520c-3p mimic than in the negative control cells. However, inhibitory effects were abolished when $3^{\prime}$-UTRs that contained both mutant-binding sites were co-transfected with miR-520c-3p, confirming that S100A4 is a target of miR-520c-3p $(P<0.01$; Fig. 5E).

Furthermore, the qRT-PCR was performed to detect the expression of miR-520c$3 p$ and S100A4 mRNA in tumor tissues and adjacent normal tissues from 128 patients suffering from PTCs. The miR-520c-3p was significantly lower in PTC tissues compared with adjacent normal tissues, and the S100A4 expression was significantly higher in PTC tissues $(P<0.01$; Fig. 5F-G). The immunohistochemistry results showed that S100A4 expression in PTC specimens was significantly upregulated compare with that in the adjacent normal tissues (93/128 VS. 25/128, $P<0.01$; Fig. 5H). Thereafter, the Pearson's correlation analysis indicated that miR-520c-3p expression level had inverse correlation with the expression of S100A4 mRNA in PTC tissues. These evidences demonstrated that S100A4 was a direct target of miR-520c-3p in PTC.

\section{Discussion}

Although the majority of PTCs usually shows an excellent prognosis after treatment, there is approximately $30 \%$ cases with lymph node metastases which prognoses unfavorable outcomes [18]. The biological behavior of PTC varies widely. However, the ideal genetic marker for PTC prognosis has not yet been identified [19]. So it is quite critical to find new biomarkers for more accurate diagnosis and treatment for PTC.

LncRNAs (long noncoding RNAs) are broadly defined as noncoding RNA molecules longer than 200 nucleotides [20,21]. They are key elements in cell differentiation and ontogenetic process through interactions with DNA, chromatin, regulatory proteins and a variety of cellular RNA species. More and more evidence has demonstrated that lncRNA have close relationship with the occurrence and development of a variety of cancers, including PTC. For example, it has been reported that lncRNA NEAT1_2 expression is up-regulated in PTC and it promotes cancer cell proliferation and tumorgenesis by functioning as a ceRNA to regulate ATAD2 expression by sponging miR-106b-5 [22]. Li et al. found that lncRNA HIT000218960 could promotes the malignant development of PTC by upregulating the expression of HMGA2 [23]. LncRNA Gas5 also has been shown to play a tumor suppressive role in PTC cells by acting as a sink for miR-222-3p, modulating the expression of PTEN [13]. HOXA-AS2, a 1048-bp lncRNA that serves as an apoptosis repressor in all-trans retinoic acid-treated NB4 promyelocytic leukemia cells, has been reported to be overexpressed in various types of malignant tumours, and promoted tumorigenesis and development [11, 24]. However, the expression of IncRNA HOXA-AS2 in PTC patients and its precise molecular mechanisms have not been completely elucidated.

In our study, we reported that HOXA-AS2 expression in PTC samples and cell lines was upregulated compared to that in surrounding non-tumor tissues as well as Nthy-ori 3-1 normal human thyroid epithelium cells. Furthermore, we found that the mechanism by which HOXA-AS2 promotes tumor progression of PTC in vitro and in vivo is mediated by inhibiting miR-520c-3p expression, thus, influenced downstream genes S100A4 expression. 
All these results supported the conclusion that HOXA-AS2 served as an oncogene in PTC to promote PTC cell proliferation.

Several studies are in agreement with our results, indicating that HOXA-AS2 affects the motility of cancer cells, which were key molecules in tumor metastasis. In this study, HOXAAS2 knockdown inhibits the migratory and invasive ability of PTC cells, indicating that high expression of HOXA-AS2 could promote PTC cell migration and invasiveness ability. HOXAAS2 might also play an important role in the regulation of EMT. HOXA-AS2 certainly increased the expression level of vimentin and $\mathrm{N}$-cadherin, and inhibit E-Cadherin expression.

The ceRNA hypothesis proposed by Pandolfi et al. suggests that endogenous RNA molecules have miRNA binding sites, which could be competitive with miRNAs, thus indirectly regulating the target gene expression of miRNAs [12]. The ceRNA hypothesis confers new and more extensive biological functions of mRNAs and non-coding RNAs. We performed a microarray to screen the HOXA-AS2-regulated miRNAs in PTC. Bioinformatics analysis (Starbase 2.0, RNA22) of miRNA recognition sequences on HOXA-AS2 revealed the presence of more than 30 miRNAs binding sites. Among them, miR-520c-3p stood out through detailed survey, which was considered to facilitate tumor accelleration effect in PTC. Luciferase assays were also used to validate the direct binding ability of the miR-520c-3p response elements on the full-length HOXA-AS2 transcript. We also confirmed that miR-520c-3p was significantly downregulated in the PTC tissues compared with the normal tissues, and miR520c-3p was a tumor suppressor gene in PTC by suppressing cell proliferation, which was consistent with results of knockdown of HOXA-AS2 expression in PTC cells.

It was well established that miRNAs function in post-transcriptional regulation mainly via directly binding to the 3'- UTR of target mRNAs, resulting in mRNA degradation or translational inhibition. To explore the potential target genes of miR-520c-3p, we used several different miRNAs target predicting software and selected out S100A4 as a downstream target of miR-520c-3p. Moreover, we further used luciferase reporting assay to illustrate S100A4 as a potential target gene of miR-520c-3p. S100A4 was previously reported to be an important protein with the capacity to promote invasion and metastasis of many human neoplasms, including PTC. In our study, the immunohistochemistry results showed that S100A4 expression in PTC specimens was significantly upregulated compare with that in the adjacent normal tissues.

\section{Conclusion}

In conclusion, this study highlights a pivotal role of HOXA-AS2 in various aspects of tumorigenesis such as proliferation, migration, invasion, and in vivo tumor growth in PTC through the post-transcriptional regulation of miR-520c-3p/S100A4 axis. Additionally, we demonstrated that silencing of HOXA-AS2 as possible mechanism during carcinogenesis, by functioning as a ceRNA to regulate ATAD2 expression by sponging miR-106b-5p. Targeting NEAT1_2 could be a promising therapeutic strategy to treat PTC.

\section{Acknowledgements}

This work was funded by the National Natural Science Foundation (NSFC) of China (81672885, 81372860) and Innovative Foundation for graduate students of Central South University (2017zzts232).

\section{Disclosure Statement}

The authors declare that no conflict of interest exists. 


\section{Cellular Physiology Cell Physiol Biochem 2018;50:1659-1672

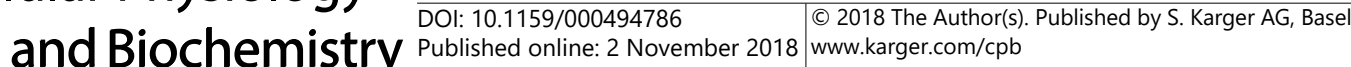

Xia et al.: HOXA-AS2 and Papillary Thyroid Cancer

\section{References}

1 Blomberg M, Feldt-Rasmussen U, Andersen KK, Kjaer SK: Thyroid cancer in Denmark 1943-2008, before and after iodine supplementation. Int J Cancer 2012;131:2360-2366.

-2 Sun L, Jiang C, Xu C, Xue H, Zhou H, Gu L, Liu Y, Xu Q: Down-regulation of long non-coding RNA RP11$708 \mathrm{H} 21.4$ is associated with poor prognosis for colorectal cancer and promotes tumorigenesis through regulating AKT/mTOR pathway. Oncotarget 2017;8:27929-27942.

-3 Frohlich E, Wahl R: The current role of targeted therapies to induce radioiodine uptake in thyroid cancer. Cancer Treat Rev 2014;40:665-674.

$>4$ Shi X, Sun M, Liu H, Yao Y, Song Y: Long non-coding RNAs: a new frontier in the study of human diseases. Cancer Lett 2013;339:159-166.

5 Ponting CP, Oliver PL, Reik W: Evolution and functions of long noncoding RNAs. Cell 2009;136:629-641.

-6 Mercer TR, Dinger ME, Mattick JS: Long non-coding RNAs: insights into functions. Nat Rev Genet 2009;10:155-159.

7 Liyanarachchi S, Li W, Yan P, Bundschuh R, Brock P, Senter L, Ringel MD, de la Chapelle A, He H: GenomeWide Expression Screening Discloses Long Noncoding RNAs Involved in Thyroid Carcinogenesis. J Clin Endocrinol Metab 2016;101:4005-4013.

-8 Zhang R, Hardin H, Huang W, Chen J, Asioli S, Righi A, Maletta F, Sapino A, Lloyd RV: MALAT1 Long Noncoding RNA Expression in Thyroid Tissues: Analysis by In Situ Hybridization and Real-Time PCR. Endocr Pathol 2017;28:7-12.

-9 Liu L, Yang J, Zhu X, Li D, Lv Z, Zhang X: Long noncoding RNA H19 competitively binds miR-17-5p to regulate YES1 expression in thyroid cancer. FEBS J 2016;283:2326-2339.

-10 Zhao H, Zhang X, Frazao JB, Condino-Neto A, Newburger PE: HOX antisense lincRNA HOXA-AS2 is an apoptosis repressor in all trans retinoic acid treated NB4 promyelocytic leukemia cells. J Cell Biochem 2013;114:2375-2383.

11 Lian Y, Li Z, Fan Y, Huang Q, Chen J, Liu W, Xiao C, Xu H: The IncRNA-HOXA-AS2/EZH2/LSD1 oncogene complex promotes cell proliferation in pancreatic cancer. Am J Transl Res 2017;9:5496-5506.

12 Salmena L, Poliseno L, Tay Y, Kats L, Pandolfi PP: A ceRNA hypothesis: the Rosetta Stone of a hidden RNA language? Cell 2011;146:353-358.

13 Wang F, Yang H, Deng Z, Su Y, Fang Q, Yin Z: HOX Antisense lincRNA HOXA-AS2 Promotes Tumorigenesis of Hepatocellular Carcinoma. Cell Physiol Biochem 2016;40:287-296.

14 Klinge CM: Non-coding RNAs: long non-coding RNAs and microRNAs in endocrine-related cancers. Endocr Relat Cancer 2018;25:R259-R282.

15 Fang Y, Wang J, Wu F, Song Y, Zhao S, Zhang Q: Long non-coding RNA HOXA-AS2 promotes proliferation and invasion of breast cancer by acting as a miR-520c-3p sponge. Oncotarget 2017;8:46090-46103.

16 Tang CP, Zhou HJ, Qin J, Luo Y, Zhang T: MicroRNA-520c-3p negatively regulates EMT by targeting IL-8 to suppress the invasion and migration of breast cancer. Oncol Rep 2017;38:3144-3152.

17 Mudduluru G, Ilm K, Fuchs S, Stein U: Epigenetic silencing of miR-520c leads to induced S100A4 expression and its mediated colorectal cancer progression. Oncotarget 2017;8:21081-21094.

-18 Hay ID, Thompson GB, Grant CS, Bergstralh EJ, Dvorak CE, Gorman CA, Maurer MS, McIver B, Mullan BP, Oberg AL, Powell CC, van Heerden JA, Goellner JR: Papillary thyroid carcinoma managed at the Mayo Clinic during six decades (1940-1999): temporal trends in initial therapy and long-term outcome in 2444 consecutively treated patients. World J Surg 2002;26:879-885.

19 Shoup M, Stojadinovic A, Nissan A, Ghossein RA, Freedman S, Brennan MF, Shah JP, Shaha AR: Prognostic indicators of outcomes in patients with distant metastases from differentiated thyroid carcinoma. J Am Coll Surg 2003;197:191-197.

20 Bian Z, Jin L, Zhang J, Yin Y, Quan C, Hu Y, Feng Y, Liu H, Fei B, Mao Y, Zhou L, Qi X, Huang S, Hua D, Xing C, Huang Z: LncRNA-UCA1 enhances cell proliferation and 5-fluorouracil resistance in colorectal cancer by inhibiting miR-204-5p. Sci Rep 2016;6:23892.

21 Weakley SM, Wang H, Yao Q Chen C: Expression and function of a large non-coding RNA gene XIST in human cancer. World J Surg 2011;35:1751-1756.

-22 Sun W, Lan X, Zhang H, Wang Z, Dong W, He L, Zhang T, Zhang P, Liu J, Qin Y: NEAT1_2 functions as a competing endogenous RNA to regulate ATAD2 expression by sponging microRNA-106b-5p in papillary thyroid cancer. Cell Death Dis 2018;9:380. 
Cellular Physiology Cell Physiol Biochem 2018:50:1659-1672 \begin{tabular}{ll|l} 
and Biochemistry & $\begin{array}{l}\text { DOI: 10.1159/000494786 } \\
\text { Published onlIne: 2 November 2018 }\end{array}$ & $\begin{array}{l}\text { C) } 2018 \text { The Author(s). Published by S. Karger AG, Basel } \\
\text { www.karger.com/cpb }\end{array}$ \\
\cline { 2 - 3 }
\end{tabular}

Xia et al.: HOXA-AS2 and Papillary Thyroid Cancer

23 Li T, Yang XD, Ye CX, Shen ZL, Yang Y, Wang B, Guo P, Gao ZD, Ye YJ, Jiang KW, Wang S: Long noncoding RNA HIT000218960 promotes papillary thyroid cancer oncogenesis and tumor progression by upregulating the expression of high mobility group AT-hook 2 (HMGA2) gene. Cell Cycle 2017;16:224-231.

-24 Xie M, Sun M, Zhu YN, Xia R, Liu YW, Ding J, Ma HW, He XZ, Zhang ZH, Liu ZJ, Liu XH, De W: Long noncoding RNA HOXA-AS2 promotes gastric cancer proliferation by epigenetically silencing P21/PLK3/DDIT3 expression. Oncotarget 2015;6:33587-33601. 